\title{
False Negative Results in Testing for Drugs of Abuse from Diluted Urine
}

\author{
Sandrine Sipouo ${ }^{1}$, Gottfried Muckel ${ }^{2}$, Bernd Huppertz $^{2}$, Michael Riedel ${ }^{3}$, \\ Ruprecht Keller ${ }^{2}$, Ralf Weiskirchen ${ }^{1 *}$ \\ ${ }^{1}$ Institute of Molecular Pathobiochemistry, Experimental Gene Therapy and Clinical Chemistry, \\ RWTH University Hospital Aachen, Aachen, Germany \\ ${ }^{2}$ Kliniken der Stadt Köln, Zentrallabor, Ostmerheimerstraße 200, 51109 Köln, Germany \\ ${ }^{3}$ Landesjustizvollzugsamt Nordrhein-Westfalen, Sedanstraße 15, 42275 Wuppertal, Germany
}

\begin{abstract}
:
Objective: The dilution of urine is a serious problem in drugs of abuse (DOA) testing. Therefore, methods and procedures that identify such adulterations are mandatory.

Method: Creatinine concentrations from 15,978 urine samples and DOA concentrations from 9,937 urine samples were determined. $11.4 \%$ of these samples were from prisoners and $88.6 \%$ from patients undergoing substitution therapy in drug substitution clinics. In our study, patients with established diagnosis for nephropathies were excluded.

Results: The mean creatinine value of $0.83 \mathrm{~g} / \mathrm{L}$ that we found in DOA samples was in the same area of healthy male individuals $(0.89 \mathrm{~g} / \mathrm{L})$. From the 9,937 DOA urine samples, 6,019 (60.57\%) were tested positive. Data from samples with diluted urine defined as $<0.2 \mathrm{~g} / \mathrm{L}$ creatinine (SAMHSA) or $<0.4 \mathrm{~g} / \mathrm{L}$ (clinical chemistry reference value) were analyzed further. A protocol for handling samples with diluted urine in the analysis of DOA is presented. The procedure takes advantage of the fact that analytical limits of quantification are lower than cutoff values of DOA analysis. The method relies on a mathematical calculation of cutoff concentrations in relation to the quotient of the urine creatinine concentration, measured in the urine specimen, and the lower reference value of creatinine in urine.
\end{abstract}

Conclusions: Using this mathematical procedure for calculation of cutoffs for diluted urine (adulteration), we could demonstrate that creatinine-dependent cutoffs are highly suitable to identify positive samples in DOA testing.

Keywords: drug testing, drugs of abuse, adulteration, creatinine, diluted urine.

Abbreviations Used: DOA, drugs of abuse; GC-MS, gas chromatography-mass spectrometry; LLOQ, lower limit of quantification; POCT, point-of-care testing; SAMHSA, Substance Abuse and Mental Health Services Administration; $\mathrm{THC}-\mathrm{COOH}$, tetrahydrocannabinol.

\section{INTRODUCTION}

Dilution of urine either by drinking excessive amounts of water ("internal dilution") or by adding water to a urine sample ("external dilution") or by taking diuretics or other special teas is a serious problem in drugs of abuse (DOA) testing [1-5]. Many subjects use this tampering method in order to dilute drugs in urine to levels that are below cutoff values in order to obtain false negative results [6]. Therefore, it is required to measure specific gravity and/or urine creatinine concentration to ensure that dilution has not occurred. Creatinine is a metabolic by-product of muscle metabolism, obligatory excreted by urine and appears in urine in relatively constant quantities with normal renal activity and normal liquid intake. Therefore, urine creatinine can be used as an indicator of urine water content or to identify a specimen as urine. The reference value in urine is $0.29-2.26 \mathrm{~g} / \mathrm{L}$ for females and 0.40 $2.78 \mathrm{~g} / \mathrm{L}$ for males [7].

The volume of urine released by a person per day varies in wide ranges depending on water intake. Therefore, a concentration of DOA in "quantity of drug/volume of urine $(\mathrm{ng} / \mathrm{mL})$ " cannot give full information on the level of drugs of the patient. For this reason, it is important to report urine 
concentrations of DOA not only as $\mathrm{ng} / \mathrm{mL}$ but also relative to the concentration of creatinine, in order to avoid false negative results by diluted samples. Cutoff values are related to DOA concentration in urine [8]. Furthermore, these values are not related to the analytical process. The cutoff for cannabinoids, for example, that is widely accepted for patients undergoing therapy in drug substitution clinics is $25 \mathrm{ng} / \mathrm{mL}$ whereas prisons in Germany have set this value to $50 \mathrm{ng} / \mathrm{mL}$. The lower limit of quantification (LLOQ) for cannabinoid detection, however, the limit of detection is about $3 \mathrm{ng} / \mathrm{mL}$ in many immunological assays $[9,10]$.

To combat the problem of diluted urine, it was suggested to use lower cutoffs for diluted urine $[4,11]$. Diluted urine was defined as a sample with a creatinine concentration of less than $0.2 \mathrm{~g} / \mathrm{L}$. For these urine samples they used the LLOQ of the cloned enzyme donor immunoassay (CEDIA) tests as cutoffs.

In the US, the Substance Abuse and Mental Health Services Administration (SAMHSA) federal workplace drug-testing program urine samples with a creatinine concentration $<0.2 \mathrm{~g} / \mathrm{L}$ are considered dilute $[8,12]$. European countries have similar guidelines given by the European Workplace Drug Testing Society (EWDTS). This value may be too low to uncover all attempts for purposely diluting urine. However, in workplace drug testing, cutoffs have been set traditionally high [12]. The rationale is that the legislation will exclude false accusations that may be very costly, especially in the litigious USA. In addition, a falsely negative tested person who might escape once will be identified as positive in DOA testing probably in another round of workplace drug testing.

Many patients and prisoners are unable to drink $1 \mathrm{~L}$ or more of water within a short timeframe. Urine from these people may have a concentration of $>0.2 \mathrm{~g}$ creatinine/L urine, although significant dilution has occurred. In addition, all creatinine concentrations which define a diluted urine specimen are somewhat arbitrary because there is a considerable variation in the excretion of creatinine based on individual patient factors, time, and method of testing [13]. Therefore, we have chosen the most common concentration limits of $0.4 \mathrm{~g} / \mathrm{L}$ as the reference in clinical chemistry reference value and $0.2 \mathrm{~g} / \mathrm{L}$ from the SAMHSA criteria of creatinine in urine as the limit to define tested urine as "diluted". Since most of the urine samples $(>95 \%)$ stem from male patients in prisons or drug substitution clinics, we generally use $0.4 \mathrm{~g} / \mathrm{L}$ as the lower reference value as defined for males.

There was criticism among doctors and officials from prisons that a sharp boundary between cutoffs of diluted and undiluted urine samples would appear to be arbitrary. Therefore, we have developed a mathematical procedure to multiply cutoff values with a variable factor that is related to the difference of the measured creatinine concentration and the lower reference value of creatinine in urine. This procedure is now implemented in drug testing programs for prisoners in the Federal State of NorthRhine Westphalia, Germany. In this workflow and in the policy of our laboratory it is, of course, selfevident to confirm positive results from screening assays with gas chromatography-mass spectrometry (GC-MS). So to sum up, the question for this study was: Is it possible to generate a mathematical procedure for calculation of cutoffs for diluted urine that allows detecting positive samples in DOA testing in screening assays.

15,978 urine samples were investigated for creatinine concentration representing the total of analyzed urine samples in the RUMA Laboratory Cologne between July 2012 and June 2013. 11,323 samples were investigated for marker substances previously described [14, 15] and 9,937 samples were investigated for DOA.

\section{MATerials AND Methods}

\subsection{Urine Samples and Ethical Issues}

15,978 urine samples were investigated for creatinine concentration that are given in $\mathrm{mmol} / \mathrm{L}$ $(1 \mathrm{mmol} / \mathrm{L}=11.31222 \mathrm{mg} / \mathrm{dL}) .11 .4 \%$ of these samples were from prisoners and $88.6 \%$ from patients undergoing substitution therapy in drug substitution clinics. Patients with established diagnosis for nephropathies were excluded from this study. In general, these patients are treated in hospitals and not in settings investigated in this study. However, extremely high concentrations of creatinine in urine (up to $7698 \mathrm{mg} / \mathrm{L}$ in women and $8886 \mathrm{mg} / \mathrm{L}$ in men) have been published in other studies that comparatively analysed approximately 45,000 urine samples from clinical chemistry and from toxicological chemistry laboratories [16]. As the samples were completely anonymized there was no 
need of any Institution Ethics Review Board for human studies. To minimize pre-analytical errors, strict precautions for collection, transport, sample preparation and analysis of urine samples were implemented [17].

\subsection{Creatinine and Drug Measurements}

For measuring creatinine concentrations in urine of hospital patients, data from 1,840 urine samples from a department of internal medicine (nephrology) that were sent to the central laboratory Cologne between January 2012 and June 2013 were investigated. Quantitative determination of creatinine in urine samples was done on a Beckman Coulter AU analyzer using the OSR6178 system reagents from Beckman Coulter. The methodology is based on a kinetic modification of the Jaffe procedure $[18,19]$ and allows determination of creatinine concentration in the range from 1-300 mg/dL. Details about specimen collection, preparation, sample storage and stability during measurement with the respective system reagent are given in the Beckman reagent package insert [20]. The tests for DOA were performed with reagents from Microgenics on an automatic Hitachi 911 analyzer from Roche as reported elsewhere [14]. Urine samples were all checked for $\mathrm{pH}$ and also occasionally for glucose, protein, haemoglobin and nitrite with a Multistix 10 SG reagent strip in an automated Clinitek Atlas automated urine chemistry analyzer system from Siemens. The laboratory offers the following test panel for DOA analysis: amphetamines, barbiturates, benzodiazepines, buprenorphine, cocaine, EDDP (2-ethylidine-1,5-dimethyl-3,3-diphenylpyrrolidine, a major metabolite of methadone), lysergic acid diethylamide (LSD), methadone, opiates, phencyclidine (PCP, "angel dust"), and tetrahydrocannabinol (THC-COOH). After the first analysis, there was a confirmation testing by GCMS for those samples, which were tested "positive" for drugs. The exact procedure was published previously [21, 22, 14].

\subsection{Data Management and Processing}

All analytical data was stored and processed in a Windows 2000-based database (Access 2007) from Microsoft. Mean values were calculated with Excel.

\subsection{Mathematical Procedure}

We applied a simple mathematical procedure for diluted urine samples $(<0.4 \mathrm{~g} / \mathrm{L})$ by which the cutoff related to the lower reference value of creatinine in urine $(0.4 \mathrm{~g} / \mathrm{L})$ following the formula:

[creatinine-dependent cutoff] $=[$ regular cutoff $(20 \mathrm{ng} / \mathrm{mL}) / 0.4] \times$ [creatinine concentration in the sample $(\mathrm{ng} / \mathrm{mL})]$.

\section{RESUltS}

\subsection{Frequency Distribution of Creatinine in Urine}

The determined distribution of creatinine concentrations in 15,978 urine samples is shown in Figure 1. The mean value of $0.83 \mathrm{~g} / \mathrm{L}$ is not significantly lower than observed from healthy male individuals $(0.89 \mathrm{~g} / \mathrm{L})$ indicating that dilution of urine is not a general phenomenon among DOA users. However, there is a significant increase of DOA positive urines in $3.16 \%$ at very low creatinine concentrations of $<0.2 \mathrm{~g} / \mathrm{L}$.

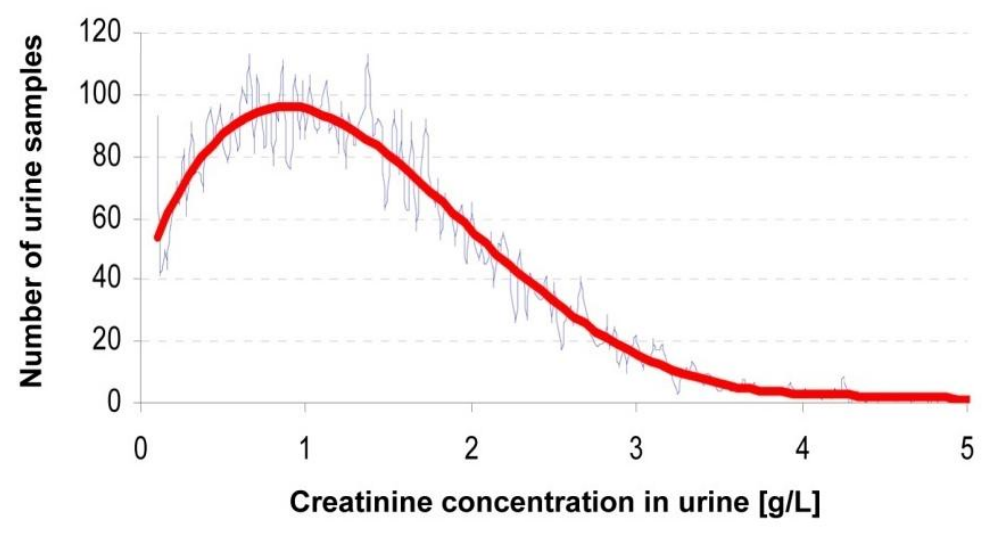

Figure1. Distribution of creatinine concentrations in urine specimen 
The creatinine concentration in urine in total 15,978 samples taken from prisons and drug substitution clinic was measured.

\subsection{Mathematical Procedures for Calculation of Cutoffs for Diluted Urine}

Among those 9,937 urine samples that were tested for DOA, 56 exhibited creatinine concentrations $<0.1 \mathrm{~g} / \mathrm{L}, 258$ samples $0.1-0.2 \mathrm{~g} / \mathrm{L}, 367$ samples $0.2-0.3 \mathrm{~g} / \mathrm{L}$ and 372 samples $0.3-0.4 \mathrm{~g} / \mathrm{L}$. Numbers of urine samples that were tested positive for DOA are given in Table 1.

Table1. Total number and percentages of urine samples relative to creatinine concentrations of 9,937 urine samples that were tested positive for DOA*

\begin{tabular}{|c|c|c|c|c|c|c|}
\hline & \multicolumn{5}{|c|}{ Concentration of creatinine in urine $[\mathrm{g} / \mathrm{L}]$} & \multirow[t]{2}{*}{ total } \\
\hline & $<0.1$ & $0.1-0.2$ & $0.2-0.3$ & 0.3-0.4 & $>0.4$ & \\
\hline Amphetamines & $0(0)^{* * *}$ & $0(0)$ & $6(4.2)$ & $4(2.9)$ & $141(2.5)$ & $151(2.5)$ \\
\hline Barbiturates & $0(0)$ & $0(0)$ & $0(0)$ & $0(0)$ & $3(0.1)$ & $3(0)$ \\
\hline Benzodiazepines & $13(52.0)$ & $25(33.8)$ & $33(23.1)$ & $25(17.9)$ & $1,230(21.8)$ & $1,326(22.0)$ \\
\hline Cannabinoids & $3(12.0)$ & $21(28.4)$ & $41(28.7)$ & $46(32.9)$ & $1,437(25.5)$ & $1,548(25.7)$ \\
\hline Cocaine & $1(4.0)$ & $3(4.1)$ & $6(4.2)$ & $4(2.9)$ & $433(7.7)$ & $447(7.4)$ \\
\hline LSD & $0(0)$ & $0(0)$ & $0(0)$ & $0(0)$ & $3(0.1)$ & $3(0)$ \\
\hline Opiates & $8(32.0)$ & $25(33.8)$ & 57 (39.9) & $61(43.6)$ & $2,390(42.4)$ & $2,541(42.2)$ \\
\hline total & 25 & 74 & 143 & 140 & 5,637 & 6,019 \\
\hline
\end{tabular}

* In our analysis, a total of 6,019 (60.57\%) from all tested samples $(9,937)$ were tested positive for DOA. ** The numbers in parentheses give the percentage (rounded to one decimal) of samples relative to the total number in the respective creatinine category that were tested positive for DOA.

382 of these diluted urine specimen still had DOA concentrations above the cutoff concentration. 429 specimens exhibited DOA concentrations between the LLOQ and cutoff. From the experience of Fraser et al. [4] and of our own laboratory, these urine samples would be regularly diagnosed "positive" in subsequent analysis on GC-MS. In our study all "positive" samples were reinvestigated with GC-MS and confirmed positive. Thus the cutoff was multiplied with a factor that was calculated from the quotient of the creatinine concentration, measured, and the creatinine concentration of the lower reference value $(0.4 \mathrm{~g} / \mathrm{L})$. Data are summarized in Table 2 . Urine samples were regarded as positive for DOA if the result of the drug test exceeded the calculated cutoff values. These samples were further investigated by GC-MS that is the confirmatory method for this purpose [12]. There is a number of 429 urine samples in the section of creatinine concentrations $<0.4 \mathrm{~g} / \mathrm{L}$ and 136 urine samples in the section of creatinine concentrations $<0.2 \mathrm{~g} / \mathrm{L}$ that would be tested "positive" (candidates for subsequent GC-MS analysis) because the concentrations of DOA is between the LLOQ of the CEDIA immunoassay and cutoff. One can calculate that only 113 (13.93\%) (in the group of creatinine $<0.4 \mathrm{~g} / \mathrm{L}$ ) or $72(30.64 \%)$ (in the group of creatinine $<0.2 \mathrm{~g} / \mathrm{L}$ ) of these urine samples would appear "positive" for DOA following our formula with a lower reference value $(0.4 \mathrm{~g} / \mathrm{L})$ for creatinine in urine. The visualization of data from urine samples that were tested for THC-COOH is shown in Figure 2.

Table2. Urine samples with creatinine $<0.4 \mathrm{~g} / \mathrm{L}$ and $<0.2 \mathrm{~g} / \mathrm{L}$

\begin{tabular}{|l|l|l|l|l|l|l|r|r|}
\hline & \multicolumn{2}{l|}{$\begin{array}{l}\text { creatinine concentration < 0.4 } \\
\text { g/L }\end{array}$} & \multicolumn{2}{l|}{$\begin{array}{l}\text { creatinine concentration < 0.2 } \\
\text { g/L }\end{array}$} & \multicolumn{2}{l|}{$\begin{array}{l}\text { analytical } \\
\text { limits [ng/mL] }\end{array}$} \\
\hline & $\begin{array}{l}\text { above } \\
\text { cutoff }\end{array}$ & $\begin{array}{l}\text { between } \\
\text { LLOQ } \\
\text { and } \\
\text { CEDIA } \\
\text { cutoff }\end{array}$ & $\begin{array}{l}\text { calculated } \\
\text { acc. to } \\
\text { formula } \\
\text { above } \\
\text { cutoff }\end{array}$ & $\begin{array}{l}\text { between } \\
\text { LLOQ } \\
\text { and } \\
\text { CEDIA } \\
\text { cutoff }\end{array}$ & $\begin{array}{l}\text { calculated } \\
\text { acc. to } \\
\text { formula }\end{array}$ & LLOQ & cutoff \\
\hline Amphetamines & 10 & 11 & $6(28.6)^{*}$ & 0 & 6 & $5(83.3)$ & 100 & 500 \\
\hline Barbiturates & 0 & 6 & $0(0)$ & 0 & 2 & $0(0)$ & 24.2 & 300 \\
\hline Benzodiazepines & 96 & 92 & $44(23.4)$ & 38 & 38 & $26(34.2)$ & 50 & 200 \\
\hline Cannabinoids & 111 & 263 & $37(9.9)$ & 24 & 74 & $28(28.6)$ & 3 & 25 \\
\hline Cocaine & 14 & 19 & $7(21.2)$ & 4 & 8 & $5(41.7)$ & 15 & 300 \\
\hline LSD & 0 & 5 & $1(20.0)$ & 0 & 1 & $1(100)$ & 0.11 & 0.5 \\
\hline Opiates & 151 & 33 & $18(9.8)$ & 33 & 7 & $7(17.5)$ & 120 & 300 \\
\hline total & $\mathbf{3 8 2}$ & $\mathbf{4 2 9}$ & $\mathbf{1 1 3 ( 1 3 . 9 )}$ & $\mathbf{9 9}$ & $\mathbf{1 3 6}$ & $\mathbf{7 2}(\mathbf{3 0 . 6})$ & & \\
\hline
\end{tabular}


Given are the total numbers of urine samples that were tested positive for DOA (above cutoff), numbers of urine samples with DOA concentrations between CEDIA LLOQ and cutoff, and numbers of positive urine samples that were calculated according to the formula given in the text. ${ }^{*}$ The numbers in parentheses give the percentage of calculated positive DOA samples (rounded to one decimal) using the mathematical procedure for calculation of cutoffs for diluted urine.
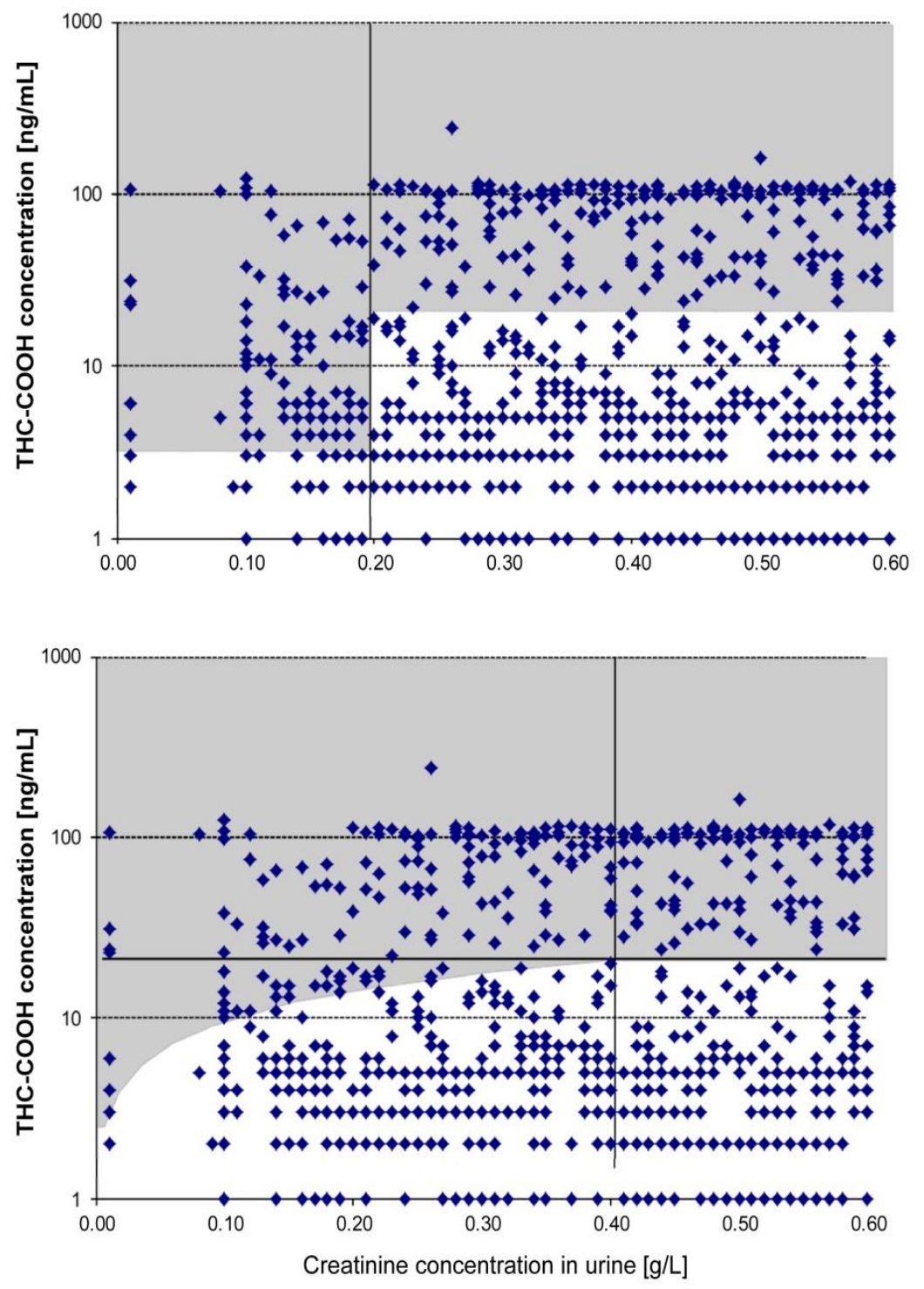

Figure2. Data from urine samples, tested for THC-COOH (cannabis)

The coloured areas are regarded "positive". Upper panel: different cutoffs for normal and diluted urines (creatinine below and above $0.2 \mathrm{~g} / \mathrm{L}$ ). Lower panel: cutoff values as calculated (for formula see text). Urine samples that are diagnosed "positive" are subject to further analysis by GC-MS.

\section{DisCUSSION}

Routine drug screening and DOA testing procedures are routinely performed in the clinical setting. In DOA screen the typical result reported is "negative" or "positive" for the presence of a specific drug suggesting that DOA testing is straightforward and easily interpretable. However, there are several hindrances that make DOA testing extremely complex and it is essential to have specialized knowledge in proper interpretation of a DOA result. Because misinterpretation of a specific result can have inappropriate consequences including unjust termination from a job, unjustifiable exclusion from a sporting event, court conflicts, and incorrect medical treatment, laboratories cannot circumvent to be involved in ethical discussions if they are active in the field of DOA testing. Therefore, the question how to handle diluted urine is not only a matter of analytical chemistry, especially when DOA testing is implemented and routinely used for quantitative screening of drugs in large sample cohorts [23]. 


\section{Sandrine Sipouo et al.}

There are many ways to handle diluted urine samples. Two examples:

- Some laboratories, in particular in area of doping controls, concentrated (spiked) urine samples or special normalization procedures that are based on specific gravity and creatinine content within the measured urine are recommended for accurate urine drug testing programs [24, 25].

- Fraser et al. use the LLOQ as the cutoff for diluted urine (creatinine in urine $<0.2 \mathrm{~g} / \mathrm{L}$ ) $[4,11]$.

Many of the problems would be solved if lower limits of detection could be used as cutoff values. Fraser et al. presented an intelligent way to take advantage of the fact that quantitative data are available from CEDIA tests at DOA concentrations below cutoffs, the method should be modified. One example: In our cohort, one patient had $5 \mathrm{ng} / \mathrm{mL}$ THC-COOH and $0.19 \mathrm{~g} / \mathrm{L}$ creatinine in his urine and was tested "positive" in GC-MS. He would have been diagnosed "negative" if the urine would have been slightly more concentrated ( $>0.21 \mathrm{~g} / \mathrm{L}$ creatinine). We believe that results on the basis of these different cutoffs for normal and diluted urine will be taken as unjustified by patients. It might also create problems in legal disputes.

Table 2 outlines that there are significant differences between urine samples declared "positive" by the Fraser approach and by our formula. The number of "positive" samples is significantly lower if our formula is applied. We have experienced that patients will rather accept an accusation of being tested positive that is based on our mathematical approach since there is not such a sharp line discriminating between diluted and undiluted samples.

Very low urine creatinine concentrations appear at various diseases such as kidney failure or cachexia. In our evaluation, we have analyzed urine samples of patients from a department of internal medicine (nephrology) of the Municipal Hospitals Cologne (data not shown). The aim was not to correlate urine creatinine concentrations to certain diagnoses, but to compare patients from drug substitution clinics and prisoners with hospital patients who are truly suffering from somatic diseases. There is a high portion of urine samples that exhibited a very low urine creatinine concentration. The frequency distribution was in general distorted to lower creatinine concentration in comparison to the curve that was observed with patients from prisons and drug substitution outpatient clinics revealing a high portion of patients $(11 \%)$ with urine creatinine $<0.2 \mathrm{~g} / \mathrm{L}$. Patients suffering from kidney diseases might claim to be treated unfairly if there would be a totally different way to handle urine samples with creatinine $<0.2 \mathrm{~g} / \mathrm{L}$. A lower reference limit of $0.2 \mathrm{~g} / \mathrm{L}$ appears to be questionable, anyway. The accepted scientific way of selecting reference values is to use the 2.5 and 97.5 percentile of the frequency distribution from concentrations of analytes.

Cutoff values, although questionable when expressed in absolute values, are widely used in the analysis of drugs of abuse. Sometimes high cutoff values are arbitrarily implemented to avoid legal problems such as THC-COOH contamination by second-hand smoking. Our formula takes this into account since we apply the cutoff values for calculation that are used in the particular setting. Therefore, cutoffs were set at a higher value. The term "clean" or "negative", therefore, with regard to DOA is not only an analytical one but also depending on the clinical setting [26].

There has been a campaign in the state of North-Rhine Westphalia, Germany, mainly among prisoners to combat drug tests in general. Many prisoners delivered urine samples that were highly diluted and "negative" if absolute cutoffs were used. This campaign culminated in two legal requests to the Ministry on Internal Affairs for generally allowing diluted urine from prisoners being tested for DOA. The protocol and its simplified flowchart that we propose in our article (Figure 3) is a possible solution for the treatment of diluted urine and the outcome of a long discussion between the laboratory and doctors from prisons and drug substitution clinics. 


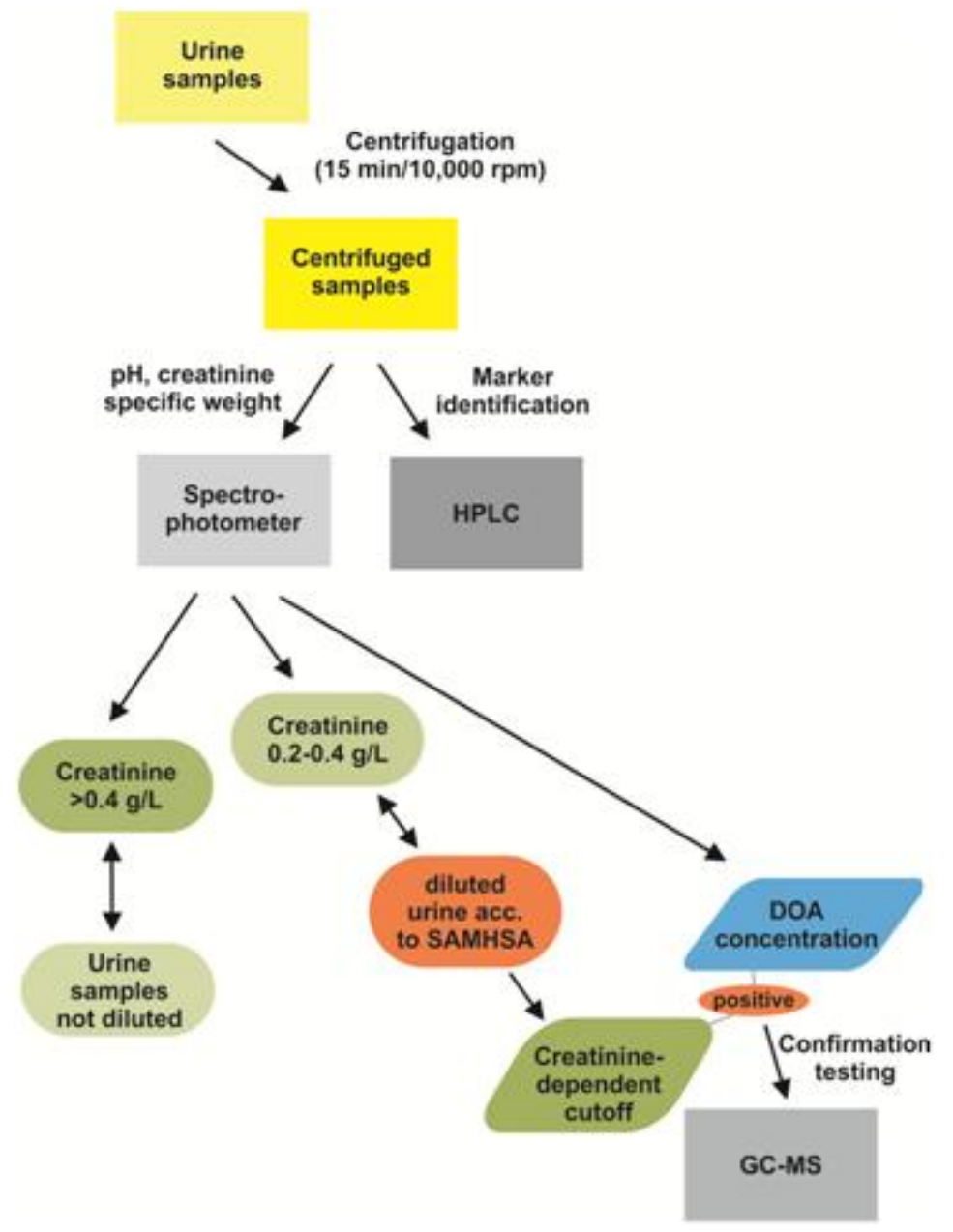

Figure3. Flowchart for testing drugs of abuse in urine

This scheme depicts the different steps in the protocol for handling samples with diluted urine in the analysis of DOA. For details, see text.

\section{Conclusion}

In summary, the mathematical procedure that we apply for the calculation of the proposed creatininedependent cutoff avoids installing a sharp boundary between the treatments of slightly and highly diluted urine. But it also may allow laboratories to offer opportunities to their customers to eliminate the tampering method of urine dilution. The mathematical procedure will add a significant diagnostic add-on that significantly impact drug screening in urine samples or DOA testing in the clinical setting, especially when cohorts (such as ours) are screened.

\section{CONTRIBUTOR SHIP}

SS, GM, BH and MR: measurements and data compiling; RK: design of the study and writing of manuscript; SS and RW: writing of manuscript.

\section{REFERENCES}

[1] Lafolie, P., Beck, O., Blennow, G., Boréus, L, Borg, S., Elwin, C. E., Karlsson, L., Odelius, G., and Hjemdahl, P. (1991). Importance of creatinine analyses of urine when screening for abused drugs. Clin. Chem., 37, 1927-1931.

[2] Needleman, S. B., Porvaznik, M., and Ander, D. (1992). Creatinine analysis in single collection urine specimens. J. Forensic. Sci., 37, 1125-1133.

[3] George, S., and Braithwaite, R. A. (1995). An investigation into the extent of possible dilution of specimens received for urinary drugs of abuse screening. Addiction, 90, 967-970.

[4] Fraser, A. D., Zamecnik, J., Keravel, J., McGrath, L, and Wells, J. (2001). Experience with urine drug testing by the Correctional Service of Canada. Forensic. Sci. Int., 121, 16-22. 
[5] Scholer, A. (2004). The effect of urine manipulation on substance abuse testing. Toxichem. Krimtech., 71, 127-137.

[6] Cone, E. J., Lange, R., and Darwin, W. D. (1998). In vivo adulteration: excess fluid ingestion causes false-negative marijuana and cocaine urine test results. J. Anal. Toxicol., 22, 460-473.

[7] Mazzachi, B. C., Peake, M. J., and Ehrhardt, V. (2000). Reference range and method comparison studies for enzymatic and Jaffé creatinine assays in plasma and serum and early morning urine. Clin. Lab., 46, 53-55.

[8] Federal Register. (2015). The daily journal of the United States Government. Mandatory Guidelines for Federal Workplace Drug Testing Programs. https://www.federalregister.gov/ articles/2015/05/15/2015-11523/mandatory-guidelines-for-federal-workplace-drug-testingprograms (accessed 10 July 2017).

[9] Mechoulam, R. (1999). Recent advantages in cannabinoid research. Forsch. Komplementarmed., 6(Suppl 3), 16-20.

[10] Stout, P. R., Horn, C. K., and Klette, K. L. (2001). Solid-phase extraction and GC-MS analysis of THC-COOH method optimized for a high-throughput forensic drug-testing laboratory. $J$. Anal. Toxicol., 25, 550-554.

[11] Fraser, A. D., and Zamecnik, J. (2003). Impact of lowering the screening and confirmation cutoff values for urine drug testing based on dilution indicators. Ther. Drug Monit., 25, 723-727.

[12] SAMHSA (2008). Substance Abuse and Mental Health Services Administration. Mandatory Guidelines for Federal Workplace Drug Testing Programs, Federal Register; Vol. 73, No 228, November 25, 2008 (FR 71858-71907). https://www.gpo.gov/fdsys/pkg/FR-2008-11-25/pdf/E826726.pdf (accessed 10 July 2017).

[13] Chu, X., Bleasby, K., Chan, G. H., Nunes, I., and Evers, R. (2016). The complexities of interpreting reversible elevated serum creatinine levels in drug development: does a correlation with inhibition of renal transporters exist? Drug Metab. Dispos., 44, 1498-1509.

[14] Huppertz, B., Gauchel, G., Feiertag, H., Schweizer, H., Krieger, H., Richter, F., Heinz, H., Blanke, J., Gastpar, M., and Keller, R. (2004). Urine labeling with orally applied marker substances in drug substitution therapy. Clin. Chem. Lab. Med., 42, 621-626.

[15] Gauchel, G., Huppertz, B., Feiertag, H., and Keller, R. (2003). Clinical use of polyethylene glycols as marker substances and determination in urine by liquid chromatography. J. Chromatogr. B Analyt. Technol. Biomed Life Sci., 787, 271-279.

[16] Arndt, T. (2009). Urine-creatinine concentration as a marker of urine dilution: Reflections using a cohort of 45,000 samples. Forensic. Sci. Int., 186, 48-51.

[17] Coppens, A., Speeckaert, M., and Delanghe, J. (2010). The pre-analytical challenges of routine urinalysis. Acta Clin. Belg., 65, 182-189.

[18] Jaffe, M. (1886). Ueber den Niederschlag, welchen Pikrinsäure in normalen Harn erzeugt und über eine neue Reaction des Kreatinins. Z. Physiol. Chem., 10, 391-400.

[19] Spencer, K. (1986). Analytical reviews in clinical biochemistry: the estimation of creatinine. Ann. Clin. Biochem., 23, 1-25.

[20] Beckman Coulter. (2010). Beckman Coulter Application Notes. https:// www. beckmancoulter. com/wsrportal/techdocs?docname=/cis/BAOSR6x78A/\%25\%25/EN_CREATININE.pdf (accessed 10 July 2017)

[21] Maurer, H. H. (1992). Systematic toxicological analysis of drugs and their metabolites by gas chromatography-mass spectrometry. J. Chromatogr., 580, 3-41.

[22] Maurer, H. H. (1999). Systematic toxicological analysis procedures for acidic drugs and/or metabolites relevant to clinical and forensic toxicology and/or doping control. J. Chromatogr B Biomed. Sci. Appl., 733, 3-25.

[23] Hegstad, S., Hermansson, S., Betnér, I., Spigset, O., and Falch, B. M. (2014). Screening and quantitative determination of drugs of abuse in diluted urine by UPLC-MS/MS. J. Chromatogr. B Analyt. Technol. Biomed. Life Sci., 947-948, 83-95. 
[24] Görgens, C., Guddat, S., Dib, J., Geyer, H. Schänzer, W., and Thevis, M. (2015). Mildronate (Meldonium) in professional sports - monitoring doping control urine samples using hydrophilic interaction liquid chromatography - high resolution/high accuracy mass spectrometry. Drug Test Anal., 7, 973-979.

[25] Cone, E. J., Caplan, Y. H., Moser, F., Robert, T., Shelby, M. K., and Black, D. L. (2009). Normalization of urinary drug concentrations with specific gravity and creatinine. J. Anal. Toxicol., 33, 1-7.

[26] World Anti-Doping Code. (2015). https://www.wada-ama.org/en/resources/the-code/world-antidoping-code (accessed 10 July 2017).

Citation: S. Sipouo et al., "False Negative Results in Testing for Drugs of Abuse from Diluted Urine", International Journal of Clinical Chemistry and Laboratory Medicine (IJCCLM), vol. 3, no. 2, p. 17, 2017. http://dx.doi.org/10.20431/2455-7153.0302002

Copyright: (C) 2017 Authors. This is an open-access article distributed under the terms of the Creative Commons Attribution License, which permits unrestricted use, distribution, and reproduction in any medium, provided the original author and source are credited. 